\title{
A New Scenario for the Formation of Massive Stellar Clusters
}

\author{
Jan Palouš \\ Astronomical Institute, Academy of Sciences of the Czech Republic, \\ Bočni II 1401, 14131 Praha 4, Czech Republic \\ Guillermo Tenorio-Tagle, Sergiy Silich \\ Instituto Nacional de Astrofísica Optica y Electrónica, AP 51, 72000 \\ Puebla, México \\ Gustavo A. Medina-Tanco \\ Instituto Astronômico e Geofísico, USP, Av. Miguel Stéfano 4200, \\ 04301-904 Sao Paulo, Brazil \\ Casiana Muñoz-Tuñon \\ Instituto de Astrofísica de Canarias, E 38200 La Laguna, Tenerife, \\ Spain
}

\begin{abstract}
A new scenario is proposed, in which the continuous creation of stars results into a highly concentrated massive (globular cluster-like) stellar system. We assume that the collapse of a massive cloud leads to the formation of first stars. Their winds and terminal supernova explosions create a standing, small radius, cold and dense shell, where the next stars form. The shell is in steady state location, which is due to a detailed balance established between the ram pressure from the collapsing cloud, the gravitational force exerted on the shell and the ram pressure of the stellar wind from the forming cluster. In this contribution we show that the standing shell remains stable against Rayleigh Taylor instability, and discuss future prospects of this work.
\end{abstract}

\section{Introduction}

Our model invokes pressure-bounded, self-gravitating, isothermal cloud, which may become gravitationally unstable if sufficiently compressed (Ebert 1955; Bonner 1956) during galaxy versus galaxy collision, when the interstellar pressure increases by several orders of magnitude above its average value. The gravitational instability allows a large cloud $\left(M_{\mathrm{c}} \sim 10^{4}-10^{6} \mathrm{M}_{\odot}\right)$ to enter its isothermal $\left(T_{\mathrm{c}} \sim 100 \mathrm{~K}\right)$ collapse phase (Larson 1969$)$, thereby developing a density and velocity structure with the following characteristics:

1. A central region of constant density (the plateau), where the infall velocity increases linearly from zero at the center, to a maximum value $v_{\max }$ at the boundary. 
2. A region of increasing size and constant maximum infall velocity, $v_{\max }$, where the density falls off as $R^{-2}$ (the skirt).

As the collapse proceeds and the density in region 1 becomes larger, the knee region in the density distribution, that separates zones 1 and 2, moves closer to the center of the configuration with an increasing speed. As the density in the plateau region increases, unstable fragments begin to form, and the first stellar generation is created in the $3 \mathrm{D}$ converging. From then onwards, stellar winds and supernova explosions will begin to have an important impact on the collapsing cloud. For this to happen however, massive stars ought to form in sufficient numbers as to jointly stop the infall at least in the most central regions of the plateau. Otherwise, individual stars, despite their mechanical energy input rate, will unavoidably be buried by the in-falling cloud, delaying the impact of feedback until more massive stars form.

\section{The Factory}

We assume that the first generation of massive stars is able to regulate itself by displacing and storing the high-density matter left over from star formation into a cool expanding shell, thereby limiting the number of sources in the first generation of stars. The shell will be driven by the momentum injected by the central wind sources and the stellar winds and radiation, which have been considered to interrupt the star formation, are trapped inside of the shell. The energy deposited by the first generation of massive stars causes the accumulation of the in-falling cloud into the standing shell, which becomes gravitationally unstable, creating the star forming factory. This model is described in greater details by Tenorio-Tagle et al. (2003).

In order to keep the shell at its standing location, the ram pressure exerted by the wind sources has to balance the in-falling gas ram pressure and the gravitational force exerted on the shell by the increasing mass of the central star cluster (see Fig. 1):

$$
4 \pi R_{\mathrm{k}}^{2} \rho_{\mathrm{w}} v_{\mathrm{w}}^{2}=4 \pi R_{\mathrm{k}}^{2} \rho_{\mathrm{k}} v_{\max }^{2}+\frac{G M_{\mathrm{sh}} M_{\mathrm{sc}}}{R_{\mathrm{k}}^{2}},
$$

where $R_{k}, \rho_{k}$ are the radius and the density at the knee and $\rho_{w}, v_{w}$ are the density and velocity of the stellar wind. The central star cluster mass is

$$
M_{\mathrm{sc}}=4 \pi R_{\mathrm{k}}^{2} \rho_{\mathrm{k}} v_{\max } t
$$

and the mass of the shell is

$$
M_{\mathrm{sh}}=4 \pi R_{\mathrm{k}}^{2} \Sigma_{\mathrm{sh}},
$$

where $\Sigma_{\mathrm{sh}}$ is the shell surface density and $t$ is the evolutionary time. Inserting (2) and (3) into eq. (1) we get

$$
\rho_{w} v_{w}^{2}=\rho_{k} v_{\max }^{2}+4 \pi G \rho_{k} \Sigma_{k} v_{\max } t
$$




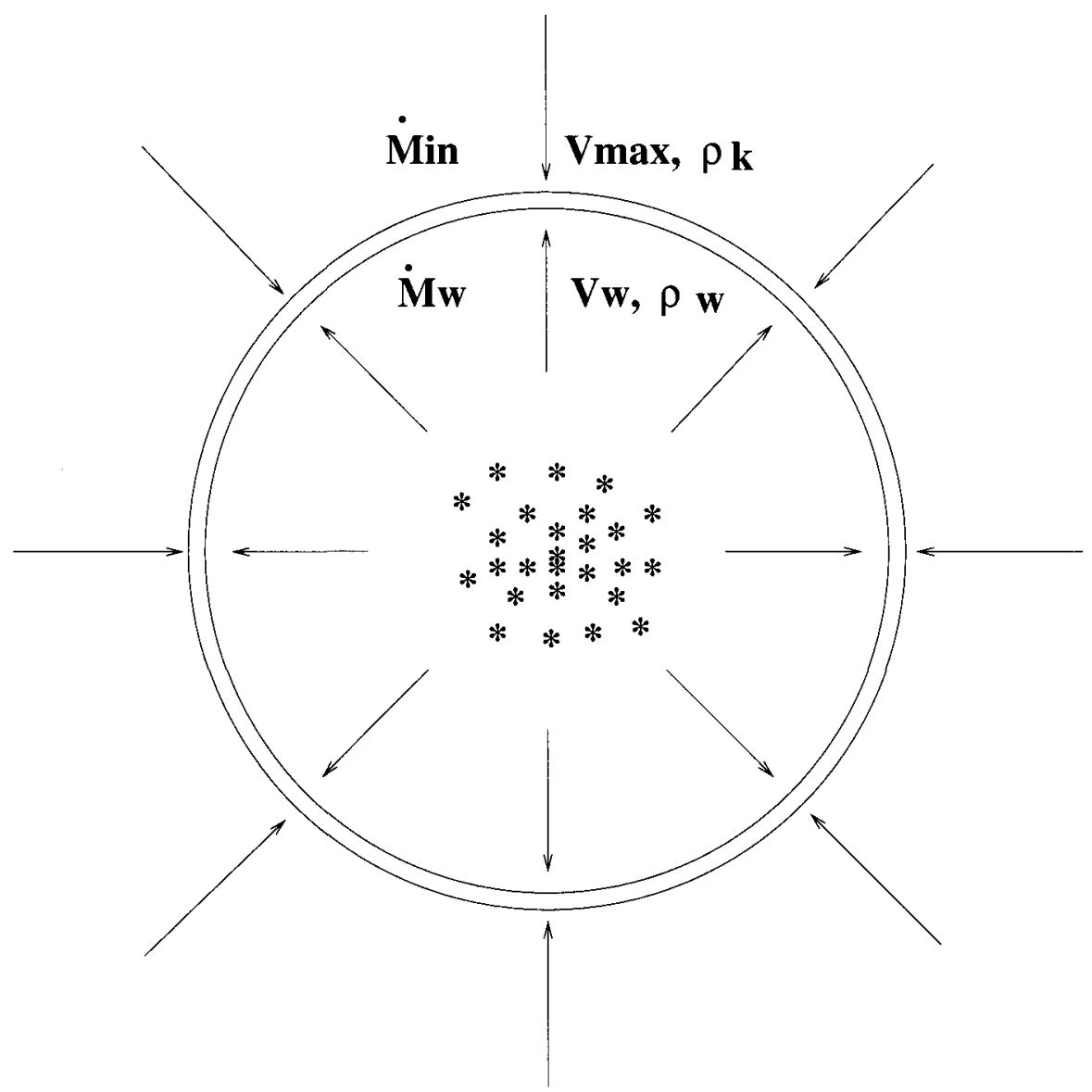

Figure 1. The standing shell inside of a collapsing cloud 
The first and the second terms on the right-hand side of Eq. (4) show that after

$$
t \geq v_{\max } /\left(4 \pi G \Sigma_{\mathrm{sh}}\right) \approx 10^{4}-10^{5} \mathrm{yr}
$$

the infall ram pressure becomes negligible compared to the gravitational pull provided by the forming cluster. Thus the shell becomes gravitationally bound with its mechanical equilibrium simply given by the equation

$$
4 \pi R_{\mathrm{k}}^{2} \rho_{\mathrm{w}} v_{\mathrm{w}}^{2}=\frac{G M_{\mathrm{sh}} M_{\mathrm{sc}}}{R_{\mathrm{k}}^{2}}
$$

The density of the shocked wind is larger than that of the shocked in-falling cloud, which shows that the shell remains stable against Rayleigh - Taylor modes.

\section{Future prospects}

The influence of the density and the velocity perturbations from spherically symmetric infall of the collapsing cloud is one issue to be solved in the future. Another important issue to be addressed is the rotation and angular momentum distribution in the collapsing cloud leading to large scale deviations from the spherical symmetry, and how this asymmetry influences the forming stellar cluster.

\section{References}

Bonner, W. B. 1956, MNRAS, 116, 351

Ebert, R. 1955, Z. Astrophys., 36, 222

Larson, R. B. 1969, MNRAS, 145, 271

Tenorio-Tagle, G., Palouš, J., Silich, S., Medina-Tanco. G. A. \& Muñoz-Tuñon, C. 2003, A\&A, 411, 397 (astro-ph/0308340) 